\title{
Evaluation of Energy-Saving \& Emission Reduction Performance of Chinese Electric Power Industry
}

\author{
Yin Chen ${ }^{1, a}$, Taihua Yang ${ }^{2, b}$ \\ ${ }^{1}$ College of Economics and Management, Shanghai University of Electric Power, Shanghai 200090, \\ China \\ ${ }^{2}$ College of Economics and Management, Shanghai University of Electric Power, Shanghai 200090, \\ China \\ akaori.sakurano@qq.com, byangth1896813@163.com
}

\begin{abstract}
Keywords: electric power industry; energy-saving \& emission reduction; performance evaluation; combination model

Abstract. Scientific performance evaluation is an important basis for government decision. This paper established the performance evaluation index system of electric power industry based on environmental technology, and then, SE-DEA model, entropy weight TOPSIS model and factor analysis model are used to calculate the scores and rankings respectively by means of the sectional data of Chinese regions in 2013. Then, Kendall-W correlation is used to verify the coherence of different evaluation results. Finally, fussy Borda model is applied to combine the independent evaluation results so that the combined evaluation result can be achieved.
\end{abstract}

\section{Introduction}

As a pillar industry of the national economy, the electric power industry consumes a large amount of energy and causes serious environmental pollution while promoting economic development and meeting the demand of electric power. In recent years, though the Chinese government has taken relevant measures, the policies of energy-saving \& emission reduction have not played a positive role in some regions. Thus, to improve the performance of energy saving \& emission reduction has become a common problem. The key to this problem is a reasonable performance evaluation which helps to provide theoretical basis for scientific and effective decision-making.

At present, the evaluation methods of environmental performance mainly include DEA [1], improved DEA [2], entropy weight TOPSIS [3], factor analysis [4] and so on. However, in order to evaluate the performance of energy saving \& emission reduction in power generation, if only one method is used, it seems one-sided and limited. So based on the single evaluation method, a kind of combination evaluation method is put forward in this paper. Moreover, the performance of energy saving \& emission reduction of China's electric power industry is evaluated and analyzed.

\section{Evaluation index system}

Less inputs, more outputs and less emissions are the ideal state of industrial production. But in fact, the desirable outputs are always accompanied by all kinds of pollution, called undesirable outputs. Given this background, [5] proposed the theory of environmental technology, which describes the relationship between inputs and 2 kinds of outputs.

Based on the environmental technology, the output sets are got, which contain the desirable outputs and the undesirable outputs. Meanwhile, the most important factors which affecting the performance of energy-saving \& emission reduction is taken into consideration. As a result, an evaluation index system can be established, shown in Fig. 1. 


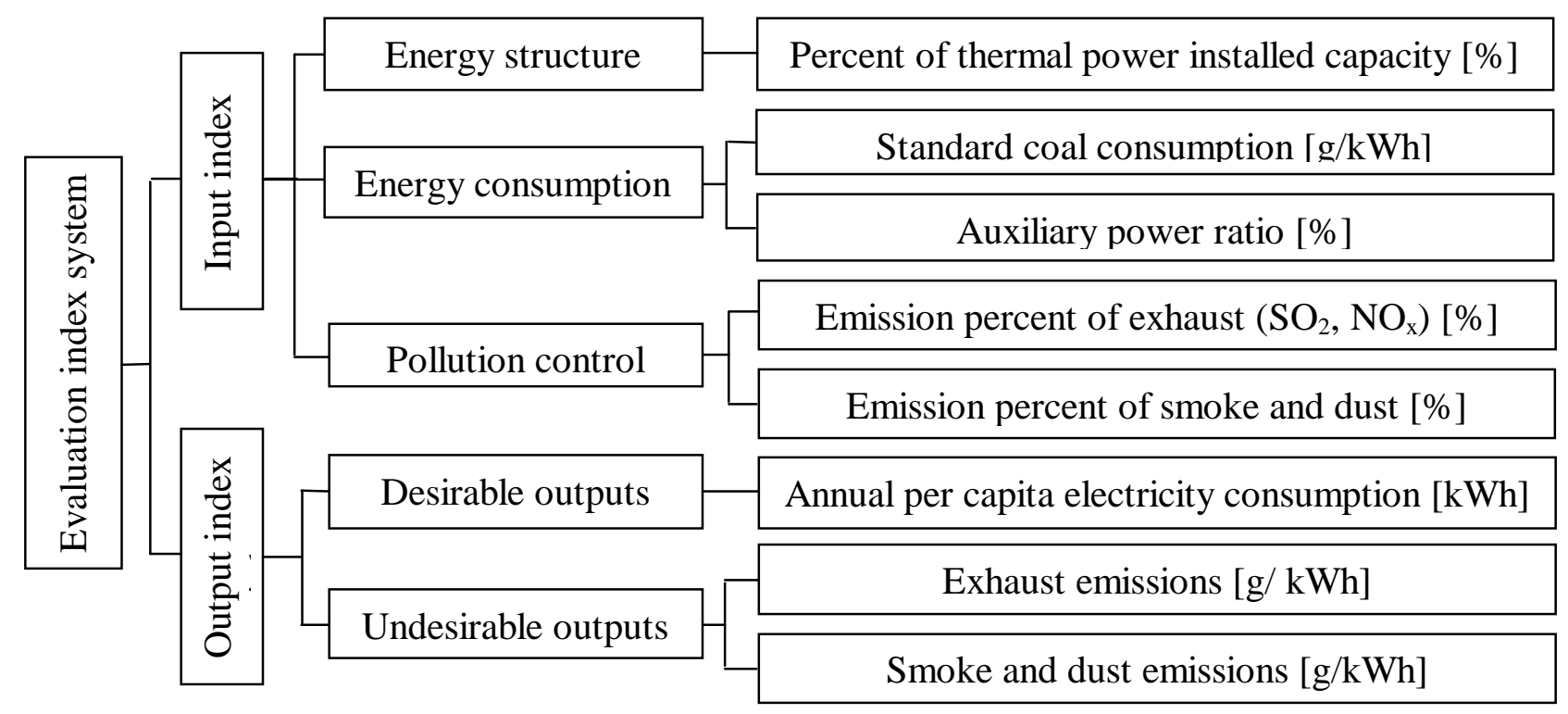

Fig. 1 Evaluation index system of energy-saving \& emission reduction performance.

For the goal of energy-saving \& emission reduction, on the one hand, energy consumption and pollution emissions should be reduced. On the other hand, the industrial structure should be adjusted so that the clean energy can achieve development. Therefore, energy consumption, pollution control and energy structure respectively corresponds to energy-saving performance, emission reduction performance and clean energy performance.

\section{Combination evaluation method}

\section{Steps of combination evaluation}

1. SE-DEA model, entropy weight TOPSIS model and factor analysis model are used to calculate the scores and rankings respectively of every region.

2. Kendall-W correlation is used to verify the coherence of different evaluation results (ex ante verification).

3. Fussy Borda model is applied to combine the independent evaluation results, through which the combined evaluation result can be got .

4. Spearman rank correlation is used to verify the coherence between individual evaluation results and combined evaluation result (ex post verification).

\section{Model calculation}

The sectional data of 30 regions (except Tibet) in 2013 is selected for calculation . Firstly, the data should be non-dimensionalized as follows.

As for input and desirable output index, let

$$
y_{i k}=0.1+0.9 \times \frac{x_{i k}-x_{\min (k)}}{x_{\max (k)}-x_{\min (k)}}
$$

as for undesirable output index, let

$$
y_{i k}=0.1+0.9 \times \frac{x_{\max (k)}-x_{i k}}{x_{\max (k)}-x_{\min (k)}}
$$

where $x_{i k}$ is the value of the $i$ th region among the $k$ th index , $x_{\max (k)}$ is the maximum value among the $k$ th index while $x_{\min (k)}$ is the minimum.

Then scores and rankings of the performances can be calculated by 3 models, shown in Table 1 . 
Table. 1 Scores and rankings of the performances calculated by single model

\begin{tabular}{|c|c|c|c|c|c|c|}
\hline \multirow{2}{*}{ Region } & \multicolumn{2}{|c|}{ SE-DEA } & \multicolumn{2}{|c|}{ Entropy weight TOPSIS } & \multicolumn{2}{|c|}{ Factor analysis } \\
\hline & Score & Ranking & Score & Ranking & Score & Ranking \\
\hline Beijing & 6.016 & 2 & 0.707 & 7 & 0.572 & 6 \\
\hline Tianjin & 1.787 & 5 & 0.692 & 13 & 0.467 & 18 \\
\hline Hebei & 0.704 & 21 & 0.661 & 18 & 0.458 & 19 \\
\hline Shanxi & 0.629 & 23 & 0.566 & 26 & 0.380 & 26 \\
\hline Inner Mongolia & 0.749 & 19 & 0.616 & 22 & 0.435 & 21 \\
\hline Liaoning & 0.549 & 27 & 0.568 & 25 & 0.388 & 25 \\
\hline Jilin & 0.535 & 28 & 0.492 & 28 & 0.343 & 29 \\
\hline Heilongjiang & 0.122 & 30 & 0.059 & 30 & 0.156 & 30 \\
\hline Shanghai & 0.869 & 15 & 0.689 & 14 & 0.511 & 14 \\
\hline Jiangsu & 0.934 & 11 & 0.705 & 8 & 0.532 & 11 \\
\hline Zhejiang & 0.920 & 12 & 0.726 & 4 & 0.550 & 8 \\
\hline Anhui & 0.914 & 13 & 0.672 & 17 & 0.497 & 15 \\
\hline Fujian & 1.155 & 8 & 0.726 & 3 & 0.576 & 5 \\
\hline Jiangxi & 0.736 & 20 & 0.647 & 20 & 0.470 & 17 \\
\hline Shandong & 0.750 & 18 & 0.658 & 19 & 0.446 & 20 \\
\hline Henan & 0.584 & 25 & 0.612 & 23 & 0.425 & 22 \\
\hline Hubei & 0.969 & 10 & 0.702 & 10 & 0.591 & 4 \\
\hline Hunan & 0.848 & 16 & 0.676 & 15 & 0.512 & 13 \\
\hline Guangdong & 0.892 & 14 & 0.715 & 5 & 0.536 & 10 \\
\hline Guangxi & 1.400 & 7 & 0.705 & 9 & 0.567 & 7 \\
\hline Hainan & 1.045 & 9 & 0.676 & 16 & 0.480 & 16 \\
\hline Chongqing & 0.485 & 29 & 0.485 & 29 & 0.352 & 28 \\
\hline Sichuan & 1.649 & 6 & 0.700 & 11 & 0.608 & 3 \\
\hline Guizhou & 0.621 & 24 & 0.549 & 27 & 0.391 & 24 \\
\hline Yunnan & 1.905 & 4 & 0.739 & 1 & 0.635 & 2 \\
\hline Shaanxi & 0.553 & 26 & 0.580 & 24 & 0.369 & 27 \\
\hline Gansu & 0.768 & 17 & 0.696 & 12 & 0.528 & 12 \\
\hline Qinghai & 7.653 & 1 & 0.713 & 6 & 0.644 & 1 \\
\hline Ningxia & 3.763 & 3 & 0.729 & 2 & 0.541 & 9 \\
\hline Xinjiang & 0.654 & 22 & 0.642 & 21 & 0.407 & 23 \\
\hline
\end{tabular}

Kendall-W correlation: $X^{2}=81.74>X_{0.025}^{2}(29)$. Verification passed.

Fuzzy Borda model strikes a balance between the scores and rankings, which makes it an advantage over other similar models in optimization [6]. By applying fuzzy Borda model to combine the independent evaluation results, the combined evaluation result can be got, shown in Table. 2.

Spearman rank correlation: $t=18.132>t_{0.025}(28)$. Verification passed.

With a standard of the scores, the 30 regions can be clustered into 4 types as follows.

Type A: Yunnan, Qinghai, Fujian, Ningxia, Beijing.

Type B: Zhejiang, Sichuan, Hubei, Guangdong, Guangxi, Jiangsu.

Type C: Gansu, Tianjin, Shanghai, Hunan, Hainan, Anhui.

Type D: Hebei, Jiangxi, Shandong, Inner Mongolia, Xinjiang, Henan, Shaanxi, Liaoning, Guizhou, Shanxi, Jilin, Chongqing, Heilongjiang. 
Table. 2 Combined evaluation result

\begin{tabular}{cccccc}
\hline Region & Score & Ranking & Region & Score & Ranking \\
\hline Beijing & 323.68 & 5 & Henan & 29.74 & 23 \\
Tianjin & 153.51 & 13 & Hubei & 271.90 & 8 \\
Hebei & 70.18 & 18 & Hunan & 131.91 & 15 \\
Shanxi & 12.00 & 27 & Guangdong & 259.53 & 9 \\
Inner Mongolia & 42.62 & 21 & Guangxi & 255.19 & 10 \\
Liaoning & 14.06 & 25 & Hainan & 119.53 & 16 \\
Jilin & 2.30 & 28 & Chongqing & 1.73 & 29 \\
Heilongjiang & 0.00 & 30 & Sichuan & 285.44 & 7 \\
Shanghai & 134.39 & 14 & Guizhou & 13.21 & 26 \\
Jiangsu & 220.94 & 11 & Yunnan & 411.13 & 1 \\
Zhejiang & 293.05 & 6 & Shaanxi & 14.68 & 24 \\
Anhui & 109.09 & 17 & Gansu & 163.56 & 12 \\
Fujian & 342.32 & 3 & Qinghai & 391.04 & 2 \\
Jiangxi & 69.12 & 19 & Ningxia & 338.79 & 4 \\
Shandong & 63.13 & 20 & Xinjiang & 38.09 & 22 \\
\hline
\end{tabular}

\section{Conclusions}

In this paper, SE-DEA model, entropy weight TOPSIS model and factor analysis model are respectively used to evaluate of China's regional energy-saving \& emission reduction performance of electric power industry. Moreover, fussy Borda model is applied to combine the independent evaluation results which can lead to the combined evaluation result.

According to the research result, the performances of different regions still have a big diversity and imbalance. Type A should process steadily on the basis of the current advantages, and provide a good demonstration effect for the others. Because of the similar development model, Type B can learn from the successful experience of Type A. Among Type C and D, the regions where the environment is suitable for the development of new energy, should take great pains to make full use of the regional advantages, while the small and medium-sized thermal power plants should be shut down. The regions where the development of new energy is limited, should take some policies of constructing major projects and suppressing small projects to fulfill the goal of structure reduction, and improve flue gas desulfurization to fulfill the goal of project reduction while exploring the way to the development of new energy.

\section{Acknowledgements}

This work was financially supported by the Shanghai Philosophy and Social Sciences Fund.

\section{References}

[1] X.P. Wang and X.H. Liu: Journal of Industrial Technological Economics Vol. 253 (2014), p. 129-135

[2] J. Liang: Industrial Economics Research Vol. 69 (2014), p. 93-102

[3] X.P. Lei and G.H. Qiu: Acta Scientiae Circumstantiae Vol. 36 (2016), p. 314-323

[4] M.Y. Cong, M.Y. Yun and J.X. Xia: Environmental Science \& Technology Vol. 37 (2014), p. 195-198

[5] R. Fare, S. Grosskopf and C.A. Pasurka: Energy Vol. 32 (2007), p. 1055-1066

[6] M. Cheng and J. Li: Operations Research and Management Science Vol. 22 (2013), p. 207-212 